\title{
Body mass index and risk of tuberculosis and death
}

\author{
Colleen F. Hanrahan ${ }^{a}$, Jonathan E. Golub ${ }^{b}$, Lerato Mohapic ${ }^{c}$, Nkeko Tshabangu ${ }^{c}$, Tebogo \\ Modisenyane $^{C}$, Richard E. Chaisson ${ }^{b}$, Glenda E. Gray ${ }^{C}$, James A. Mclntyre ${ }^{C}$, and Neil A. \\ Martinson ${ }^{b, c}$ \\ a Johns Hopkins Bloomberg School of Public Heath, Baltimore, Maryland, USA \\ b Johns Hopkins University Center for TB Research, Baltimore, Maryland, USA \\ c Perinatal HIV Research Unit, University of the Witwatersrand, Johannesburg, South Africa
}

\section{Abstract}

Background-High BMI has been shown to be protective against tuberculosis (TB) among HIV-uninfected individuals, as well as against disease progression and mortality among those with HIV. We examined the effect of BMI on all-cause mortality and TB incidence among a cohort of HIV-infected adults in Soweto, South Africa.

Methods-A clinical cohort of $3456 \mathrm{HIV}$-infected adults from South Africa was prospectively followed from 2003 to 2008 with regular monitoring. The primary exposure was BMI and the outcomes of interest were all-cause mortality and a newly diagnosed episode of TB. Cox proportional hazard models assessed associations with risk of mortality or incident TB.

Results-Incidence rates of mortality were $10.4 / 100$ person-years for baseline BMI of 18.5 or less, 3.6/100 person-years for baseline BMI 18.6-25, 1.7/100 person-years for baseline BMI 25.130 , and 1.6/100 person-years for baseline BMI more than 30. Compared to those with normal BMI, overweight and obese participants had a significantly reduced risk of mortality [adjusted hazard ratio 0.59 (95\% confidence interval, CI 0.40-0.87) and 0.48 (95\% CI 0.29-0.80), respectively]. Incidence rates of TB by baseline BMI were 7.3/100 person-years for underweight, 6.0/100 person-years for normal, 3.2/100 person-years for overweight, and 1.9/100 person-years for obese. Compared to those with normal BMI, those with overweight and obese BMI were at a significantly reduced risk of developing TB [adjusted hazard ratio 0.56 (95\% CI 0.38-0.83) and 0.33 (95\% CI 0.19-0.55), respectively].

Conclusion-HIV-infected individuals with obese and overweight BMI have a significantly reduced risk of both mortality and TB, after adjusting for HAART use and CD4 cell count.

\section{Keywords}

body mass index; HAART; HIV; mortality; nutrition; tuberculosis

\section{Background}

Currently, there are 33 million people worldwide infected with HIV and South Africa bears the largest global burden of this disease, with an estimated 5.7 million infected individuals [1]. Despite the rollout of HAART in South Africa, tuberculosis (TB) incidence (940/100 000/years) continues to rise, as does mortality due to TB (218/100 000/years) [2]. TB remains the leading cause of death among those with HIV in Africa [1].

Correspondence to Dr Neil A. Martinson, Perinatal HIV Research Unit, PO Box 144, Diepkloof 1864, Johannesburg, South Africa. Tel: +27 119899703; fax: +27 119899762; Neil1.martinson@gmail.com. 
Low BMI is a strong, independent predictor of mortality among HIV-infected adults [3,4], a relationship also found in those initiating HAART [5,6]. Overweight and obese BMI, traditionally associated with increased mortality, have been shown to be protective against mortality and disease progression in HIV-infected individuals [7-9]. No published studies to date have examined the relationship between overweight/obese BMI and mortality or risk of TB set in the context of a developing country. As one of the most developed countries in sub-Saharan Africa, South Africa has a rising prevalence of obesity. The most recent Demographic and Health Survey reported that $21 \%$ of men were overweight and $7 \%$ were obese, whereas among women $27 \%$ were overweight and $27 \%$ were obese [10]. A survey conducted in the KwaZulu-Natal Province of South Africa found 43 and 17\% prevalence of overweight and obesity, respectively, among HIV-infected individuals [11].

We used prospectively collected data from a large cohort of HIV-infected adults in Soweto, South Africa to investigate the relationship between BMI and both all-cause mortality and incident TB.

\section{Methods}

The Perinatal HIV Research Unit (PHRU) operates a wellness clinic, which has provided preantiretroviral therapy (pre-ART) care and support for HIV-infected adults in Soweto, South Africa since 2003. Since HAART became available in April 2004, patients meeting treatment criteria are referred for HAART initiation; where possible they are followed up while taking HAART. Soweto is a relatively well resourced suburb of Johannesburg with high rates of home electrification and access to piped potable water. The median GDP per capita in Gauteng province where Soweto is located is approximately USD 5000 [12] and antenatal HIV seroprevalence is approximately $30 \%$ [13].

Participants in this cohort study were either self-referred or identified through voluntary counseling and testing programs or research programs at the PHRU. Consenting adults over the age of 18 were enrolled from July 2003 to December 2008. A questionnaire covering sociodemographics, clinical symptoms, and general medical history was administered by a study nurse at the initial visit. Ongoing data collection visits were scheduled 4-7 months apart during which a follow-up questionnaire was administered, collecting inter alia information on hospital admissions, TB symptoms, HAART use and compliance, and use of other medications. Weight was measured and CD4 cell counts were determined at each study visit. Height was measured at baseline. At least one sputum sample was collected from TB suspects for smear microscopy and liquid culture. Patients were offered cotrimoxazole according to modified WHO guidelines. Isoniazid preventive therapy (IPT) was offered to patients with a positive tuberculin skin test (TST) until January 2009, after which it was offered to all those without a history of prior TB as well as no signs of active TB. Family planning methods were offered to women, and symptomatic patients were provided with syndromic sexually transmitted infection treatment, and when appropriate, treatment for common HIV-related conditions. Referrals were made for HAART when patients became eligible according to the then current South African guidelines (CD4 cell count $<200$ cells/ $\mu 1$ or WHO stage 4 illness) [14].

Patients not returning for study visits were actively followed through phone calls, reminders mailed to their addresses, and home visits. Deaths were ascertained through family contacts or home visits and were verified by death certificate whenever available. Ethics approval to collect data was obtained from University of the Witwatersrand's Human Research Ethics Committee and for secondary data analysis without personal identifiers, from the Johns Hopkins Medicine institutional review board. 


\section{Exposure variables}

BMI $\left(\mathrm{kg} / \mathrm{m}^{2}\right)$ was investigated as both a baseline and time-varying covariate in separate analyses and was categorized according to the WHO International Classification of Adult BMI as follows: underweight (BMI $\leq 18.5$ ), normal (BMI 18.6-25), overweight (BMI 25.130), and obese (BMI >30) [15].

CD4 cell count and complete blood count was assessed by flow cytometry. CD4 cell count was investigated as both a baseline and time-varying covariate in separate analyses and was

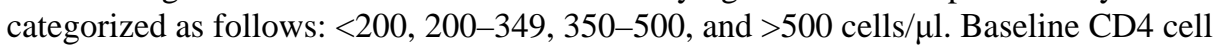
count values were restricted to those within 1 year of baseline visit, whereas those for most recent visit were restricted to those within 6 months of each follow-up visit. HAART and cotrimoxazole use was treated as a time-varying covariate, but once initiated, was assumed to continue uninterrupted until censoring or death. IPT use was treated as time-varying.

Individuals reporting an episode of TB occurring more than 9 months prior to the first study visit were considered to have a history of TB. Individuals with either an episode of TB that occurred within the 9 months prior to the first study visit, one diagnosed at the first study visit, or one within 60 days after the first study visit were considered to have prevalent TB at baseline. Baseline household income was categorized as follows: $<1000$ Rand/month, 1000-5000 Rand/month, and > 5000 Rand/month.

\section{Outcome variables}

Time to death was calculated from the date of baseline visit to reported date of death. A TB case was defined as the first instance of any one of the following: recorded initiation of multidrug TB therapy, presence of acid fast bacilli on microscopy or a biopsy suggestive of $\mathrm{TB}$, mycobacterial culture positive for acid fast bacilli or Mycobacterium tuberculosis, being admitted to hospital with a diagnosis of TB, or cause of death ascribed to TB. TB cases were captured from among those found at regular visits, self-reported hospital admissions, and death certificates. Time to incident TB was recorded from the date of baseline visit to date of first occurrence of TB for those without TB at the baseline visit. Individuals with prevalent TB at baseline were only included in the analysis 270 days after their baseline visit, to allow for completion of treatment of TB during which they were not at risk for another episode of TB. Individuals who did not die or experience an event of interest in each analysis were censored at their final study visit.

\section{Statistical methods}

All statistical analyses were conducted using Stata/SE 10.1 [16]. Multiple imputation was used to assign values for the 7\% (265) of participants missing values for height. The model used for imputing height was a linear model conditional on sex. Five separate imputed datasets were created [17]. Baseline values of BMI (Table 1) are reported using the mean value of these imputed heights, whereas those in the Cox proportional hazard model results (Tables 2 and 3) are reported using Rubin's pooling method [17]. The Stata ICE package was used to perform multiple imputation [18]. Confidence intervals (CIs) for binomial proportions were calculated using the Wald technique and the log-rank statistic was used to compare Kaplan-Meier curves. Cox proportional hazard models were used for all survival analyses, and the proportional hazard assumptions were checked using Schoenfeld residuals. Statistical significance was set at 0.05 for all analyses.

Associations between BMI and each outcome were investigated in several ways. First, we assessed associations of BMI and the outcomes of mortality and TB by using the BMI at entry to the cohort. Second, to ensure temporality, BMI was included as a time-varying variable, but lagged by one study visit to ascertain the most recent BMI prior to an event. 
Additionally, we performed several sensitivity analyses: excluding participants who had lost more than $10 \%$ of their body weight between the first and second visit (according to the WHO definition of wasting [19]) from the analyses; excluding those with TB at baseline from the mortality analysis; and excluding those weighing less than $45 \mathrm{~kg}$ at baseline from both the mortality and TB analyses.

\section{Results}

A total of 3635 HIV-infected individuals were enrolled at the PHRU wellness clinic between June 2003 and May 2009. One hundred thirty (3\%) were excluded from the analysis due to missing baseline CD4 cell count, an additional 46 (2\%) due to missing baseline weight, and three $(<1 \%)$ due to missing date of birth. Two hundred sixty-five (7\%) individuals for whom height was imputed did not differ significantly from the remaining cohort on any characteristic of interest in this study (data not shown). The baseline characteristics for the remaining 3456 individuals at entry into the cohort stratified by baseline BMI are shown in Table 1. At baseline, $41.1 \%$ (1419) of all individuals were overweight or obese (95\% CI $39.4-42.8), 49.2 \%$ (1702) had normal BMI (95\% CI 47.6-50.9\%), and 9.6\% (334) were underweight (95\% CI 8.7-10.7\%). More women were obese and overweight than men at baseline; $26.4 \%$ (715) of women compared to $11.0 \%$ (83) of men were overweight ( $P$ $<0.001$ ) and $22.2 \%$ (599) of women compared to $2.9 \%$ (22) of men were obese $(P<0.001)$. During follow-up, 782 individuals (22.6\%) were started on HAART, with a median CD4 cell count at HAART initiation of 138 cells/ $\mu 1$ [interquartile range (IQR) 65-190]. Four hundred and eighty (13.9\%) individuals initiated IPT. The loss to follow-up by 2 years was $9.0 \%$ (95\% CI 8.1-10.0). Those lost to follow-up did not differ significantly from those not lost to follow-up by any characteristic of interest in this study, including age, sex, baseline CD4 cell count, baseline BMI, or baseline household income. Median follow-up time by baseline BMI category was 2.9 years (IQR 2.1-3.8) for underweight; 3.2 years (IQR 2.43.9) for normal; 3.2 years (IQR 2.4-3.8) for overweight; and 3.2 years (IQR 2.3-3.8) for obese.

\section{Mortality}

Three thousand, four hundred and fifty-six patients had 8038 person-years of follow-up, during which 280 deaths were recorded: $3.5 / 100$ person-years (95\% CI 3.1-3.9). The median age at death was 35.4 years (IQR 31.0-40.7). The median BMI at baseline visit of those who died and at the visit prior to death was $20.8 \mathrm{~kg} / \mathrm{m}^{2}$ (IQR 18.4-23.6) and $20.5 \mathrm{~kg}$ / $\mathrm{m}^{2}$ (IQR 18.2-23.1), respectively. Likewise, median CD4 cell counts at baseline visit of those who died and at the visit prior to death were 123 cells/ $\mu \mathrm{l}$ (IQR 43-247) and 89 cells/ $\mu \mathrm{l}$ (IQR 28-173). A total of 7.9\% (95\% CI 5.0-11.7) of all deaths occurred while the individual was on HAART. In these, the median time from HAART initiation to death was 10 months (IQR 7-17).

In the underweight, normal, overweight, and obese categories, there were 80 deaths, 10.4/100 person-years (95\% CI 8.3-12.9); 146 deaths, 3.6/100 person-years (95\% CI 3.14.7); 31 deaths, $1.7 / 100$ person-years (95\% CI 1.2-2.4); and 23 deaths, $1.6 / 100$ person-years (95\% CI 1.1-2.4), respectively (Fig. 1). Mortality rates by most recent BMI were similar to those at baseline BMI (data not shown). The median time between most recent BMI and death was 5.7 months (IQR 3.2-9.9). Figure 2 presents the Kaplan-Meier survival curves stratified by baseline BMI.

Using a Cox proportional hazard model, individuals with either overweight or obese BMI at baseline had a significantly reduced risk of mortality compared to those with normal BMI at baseline [adjusted hazard ratio (aHR), 0.55 (95\% CI 0.37-0.82) and 0.60 (95\% CI $0.38-$ 0.95), respectively], after adjusting for baseline CD4 cell count, time-varying HAART use, 
cotrimoxazole use, sex, age, household income, employment, baseline smoking status, and years since HIV diagnosis. These findings were unchanged after excluding 135 (3.9\%) individuals whose body mass decreased more than $10 \%$ between their first two visits; excluding 155 (4.4\%) individuals with a baseline weight less than $45 \mathrm{~kg}$; and dropping 281 individuals with prevalent TB at baseline (8.1\%; data not shown).

A Cox proportional hazard model was also used to investigate risk of death in each most recent BMI category, relative to the normal (18.5-25) BMI category (Table 2). Those in the overweight and obese categories had a significantly reduced risk of death compared to those in the normal category [aHR 0.59 (95\% CI\% CI 0.40-0.87) and 0.48 (95\% CI 0.29-0.80), respectively]. This relationship held in the fully adjusted model after accounting for HAART use, measures of disease severity (most recent CD4 cell count and years since HIV diagnosis), socioeconomic status (household income at baseline, employment status) as well as cotrimoxazole use, age, sex, and baseline smoking status.

\section{Tuberculosis}

At baseline, 681 (20\%; 95\% CI 18-21) patients reported an episode of TB occurring more than 9 months before their first study visit. The prevalence of TB at the baseline visit was $8 \%$ (95\% CI 7-9). Three thousand, four hundred and fifty-six patients had a total of 5000 person-years of follow-up for TB during which 226 incident TB cases occurred; 4.5/100 person-years (95\% CI 4.0-5.1). Incidence rates for each baseline BMI category were as follows: underweight, 7.3/100 person-years (95\% CI 4.9-10.9); normal, 6.0/100 personyears (95\% CI 5.1-7.0); overweight, 3.2/100 person-years (95\% CI 2.4-4.4); and obese, 1.9/100 person-years (95\% CI 1.2-3.0; Fig. 1). TB incidence among those not on HAART was $4.7 / 100$ person-years (95\% CI 4.0-5.8) and for those on HAART was 4.1/100 personyears (95\% CI 3.2-5.3). Incidence rates by most recent BMI category were similar to those by baseline BMI (data not shown). The median CD4 cell count at diagnosis of incident TB was 169 cells/ $\mu \mathrm{l}$ (IQR 88-276). Figure 2 presents the Kaplan-Meier survival curves for TB stratified by baseline BMI.

Using a Cox proportional hazard model, individuals with overweight and obese BMI at baseline had a significantly reduced risk of incident TB compared to those with normal baseline BMI [aHR 0.61 (95\% CI 0.42-0.88) and 0.36 (95\% CI 0.22-0.60), respectively], after adjusting for baseline CD4 cell count, HAART use, history of TB, IPT use, sex, age, household income, employment status, baseline smoking status, and years since HIV diagnosis.

These results remained significant and unchanged after excluding 681 individuals with either prevalent TB or a history of TB. Further excluding 135 (4\%) patients who lost more than $10 \%$ of their body weight between the first two visits also did not significantly alter the effect of baseline BMI category on incident TB within this cohort, nor did exclusion of 155 (5\%) individuals with a baseline weight less than $45 \mathrm{~kg}$.

A Cox proportional hazard model was also used to investigate the relationship between most recent BMI and incident TB within the cohort, and Table 3 shows the univariate and aHR for risk of TB within each BMI category, relative to normal BMI. The multivariate model was adjusted for CD4 cell count at most recent visit, time-varying HAART, history of TB, IPT use, sex, age, household income, employment status, baseline smoking status, and years since HIV diagnosis. Similar to mortality, those in the overweight and obese categories had significantly decreased hazards of TB [aHR 0.56 (95\% CI 0.38-0.83) and 0.33 (95\% CI $0.19-0.55)$, respectively] compared to those with normal BMI. The median time between an incident TB case and most recent BMI measurement was 4.1 months (IQR 2.6-6.1).

Excluding those with a history of TB resulted in similar inferences for overweight and obese 
compared to normal [aHR 0.49 (95\% CI 0.31-0.79) and 0.29 (95\% CI 0.16-0.54), respectively].

\section{Discussion}

Our findings show a clear protective effect, with a dose response, of increasing BMI on both all-cause mortality and incident TB among HIV-infected adults in a South African cohort, after adjusting for CD4 cell count and HAART use. Specifically, persons with obese and overweight BMI have a significantly decreased risk of both mortality and TB, whereas those with underweight BMI have an increased risk of mortality. Our results are consistent with those of several published studies. Shor-Posner et al. [8] reported an inverse relationship between BMI and mortality, independent of baseline CD4 cell count in a cohort of HIVpositive intravenous drug users in Miami, and Shuter et al. [9] found that overweight individuals had slower disease progression and lower viral load among a cohort of AIDSfree HIV-positive individuals in New York City, after adjusting for baseline CD4 cell count and time to antiretroviral initiation. Among a multisite US cohort of HIV-positive women, Jones et al. [7] found that both higher baseline and time-varying BMI were associated with slower disease progression, lower risk of clinical AIDS, and decreased risk of HIV-related mortality, after adjusting for CD4 cell count and viral load.

As in our study, individuals with obese and overweight BMI were also recently shown to be at a lower risk of TB, as compared to those with normal and underweight BMI, in a large cohort of elderly health-center patients in Hong Kong [20]. HIV status was unreported in this study, but given the demographic characteristics of the population under study, it can be assumed to have been low. This finding confirmed two earlier large studies, one conducted by Tverdal in Norway in 1986 [21] showing a decreased risk of TB with increasing BMI and one by Edwards et al. in 1971 [22], finding a decreased progression to active TB disease among US naval recruits with an 'overweight body build' compared to those who were underweight. Recently, in Mumbai, India overweight men and women were shown to be at decreased risk for death attributed to TB [23].

Nutritional differences between overweight or obese individuals and those with normal weight may be one biological driver for the protective effect seen here against both mortality and TB. Individuals with increased BMI may have higher daily protein and energy intake, which could result in more robust immune function and drive a reduction in both mortality and TB. However, a recent meta-analysis of eight randomized trials of macronutrient supplementation in HIV-infected adults found no increase in CD4 cell count, nor any evidence that supplemented protein and energy intake had an effect on mortality among these individuals [24].

Differences in micronutrient intake could also be implicated in the associations shown here, as many micronutrient deficiencies have been described among those with TB as well as HIV [25-29], but randomized trials of micronutrient supplementation for individuals with HIV have shown only modest effects on disease progression, morbidity, and mortality, with vitamin A alone showing an effect on all-cause mortality, and this only in children [30]. Vitamin D deficiency has been implicated as a risk factor for the development of TB, though a recent supplementation trial showed no clinical impact [31]. Although apparently protective against TB and all-cause mortality, a high proportion of these HIV-infected adults were overweight, raising concerns about current and future risks of metabolic and cardiovascular adverse health consequences, particularly those which may be exacerbated by long-term HAART use. 
Overall, our study confirms the effectiveness of HAART in reducing both mortality and incident TB among HIV-positive individuals in sub-Saharan Africa [32,33]. Although crude TB incidence rates did not vary significantly between those on and off HAART, after adjusting for most recent CD4 cell count, a significant effect of HAART on reduction in TB incidence is seen. The relative health of this wellness cohort as evidenced by a high median CD 4 cell count at HAART initiation ( 185 cells/ $\mu$ l) could explain the absence of high mortality within the first 6 months of HAART as seen by other studies in South Africa $[34,35]$.

This study has several limitations. First, it was conducted using a clinical cohort relying on self-report for many exposure variables. The overall mortality rate reported here (3.5\%) suggests an incomplete reporting of deaths among the cohort and that a portion of those lost to follow-up are likely misclassified deaths; however, there is no reason to believe that such misclassification would occur differentially by participant BMI. Temporality of exposure and outcome are also an issue in this study, particularly in looking at TB, of which weight loss is one classic symptom. We attempted to minimize these effects to causal inference by examining the effect of BMI both as a baseline exposure and a time-varying one, lagged by one visit in order to ensure that the exposure period preceded the outcome diagnosis. Additionally, we repeated our analyses removing individuals who exhibited signs of wasting and, thus, may have been undiagnosed cases of TB at entry to the cohort, as well as individuals who had TB at their baseline visit without finding a change in our inferences about the association between BMI and development of TB. Residual confounding due to unmeasured factors - particularly socioeconomic - cannot be excluded, though we did include employment and household income in our multivariate models. A simple 'intention to treat' approach was used to examine the effect of cotrimoxazole use on mortality within this cohort, which could explain the attenuated effect size [aHR 0.84 (95\% CI 0.65-1.1)]. A more in-depth analysis utilizing marginal structural models to appropriately handle confounding by indication and time-varying confounding affected by prior exposure may be more suitable to explore the influence of cotrimoxazole on mortality. Finally, we have no measures of viral load, dietary intake, or plasma micronutrient levels, making it difficult to identify a single protective factor. Additional body composition measures and nutritional assessments would add to our limited understanding of the biological mechanisms at work behind these findings. Nevertheless, our study's strength is that it includes several thousand HIV-infected adults using easily measured anthropomorphic measures in a cohort from a relatively well resourced setting with access to both cotrimoxazole and HAART.

Though this study does not attempt to elucidate the biological mechanisms behind the protective effect of obese and overweight BMI against TB and mortality, it does provide rationale for further studies to do so. Our study provides evidence that although it is a relatively crude measure, BMI may be a useful surrogate marker of risk of TB or death among HIV-positive individuals, but urgent studies are required to pinpoint the protective factor and to address detrimental health issues that may result from elevated BMI.

\section{Acknowledgments}

The authors are grateful to the HIV-infected men and women whose data were used in this study, the primary healthcare nurses, and other team members who provide care and treatment; Dr Melamu who provides expert assistance and support to the nurses; Yudesh Baliram who manages the data; and Helen Struthers for grant administration and support.

C.H. conceived the study, did the analyses with assistance from J.E.G. and N.A.M., and with N.A.M. and J.E.G. wrote the paper. T.M. cleaned and formatted data and did preliminary analyses. R.E.C., J.A.M., and G.E.G. assisted in the design and implementation of the cohort and this study and assisted with drafting the article. L.M. provided patient care and collected data. N.T. managed the study. All authors had opportunity to review the submitted article. 
Patient care is funded by a US PEPFAR grant through USAID South Africa (674-A-00-08-00009-00). NIH grants AI066994, AI001637, HL090312, and AI048526 supported the analysis. T.M. received research training through Fogarty International Center Grants (2RTW007370/3). C.H. received travel support through an award from the Johns Hopkins Center for Global Health. The opinions herein do not necessarily reflect those of USAID or the US Government.

\section{References}

1. UNAIDS. AIDS epidemic update 2009. Geneva: WHO; 2009. http://data.unaids.org/pub/Report/2009/JC1700_Epi_Update_2009_en.pdf

2. WHO. Global tuberculosis control: epidemiology, strategy, financing. Geneva: WHO; 2009. http://www.who.int/tb/publications/global_report/2009/pdf/full_report.pdf

3. Lindan CP, Allen S, Serufilira A, Lifson AR, Van de Perre P, Chen-Rundle A, et al. Predictors of mortality among HIV-infected women in Kigali, Rwanda. Ann Intern Med. 1992; 122:320-328. [PubMed: 1733389]

4. Van der Sande MA, Schim van der Loeff MF, Aveika AA, Sabally S, Togun T, Sarge-Njie R, et al. Body mass index at time of HIV diagnosis: a strong and independent predictor of survival. J Acquir Immune Defic Syndr. 2004; 7:1288-1294. [PubMed: 15385737]

5. Moh R, Danel C, Messou E, Ouassa T, Gabillard D, Arizian A, et al. Incidence and determinants of mortality and morbidity following early antiretroviral therapy initiation in HIV-infected adults in west Africa. AIDS. 2007; 21:2483-2491. [PubMed: 18025885]

6. Zachariah R, Fitzgerald M, Massaquoi M, Pasulani O, Arnould L, Makombe S, et al. Risk factors for high early mortality in patients on antiretroviral treatment in a rural district of Malawi. AIDS. 2006; 20:2355-2360. [PubMed: 17117022]

7. Jones CY, Hogan JW, Snyder B, Klein RS, Rompalo A, Schuman P, et al. Overweight and human immunodeficiency virus (HIV) progression and changes in body mass index in women in the HIV epidemiology research study cohort. Clin Infect Dis. 2003; 37 (Suppl 2):S69-S80. [PubMed: 12942377]

8. Shor-Posner G, Campa A, Zhang G, Persaud N, Miguez-Burbano MJ, Fletcher MA, et al. When obesity is desirable: a longitudinal study of the Miamia HIV-1 infected drug abusers (MIDAS) cohort. J Acquir Immune Defic Syndr. 2000; 23:81-88. [PubMed: 10708060]

9. Shuter J, Chang CJ, Klein RS. Prevalence and predictive value of overweight in an urban HIV care clinic. J Acquir Immune Defic Syndr. 2001; 26:291-297. [PubMed: 11242203]

10. South African Department of Health, Medical Research Council, OrcMacro. South Africa Demographic and Health Survey 2003. Pretoria: Department of Health; 2003. http://www.mrc.ac.za/bod/sadhs.htm

11. Bärnighausen T, Welz T, Hosegood V, Batsing-Feigenbaum J, Tanser F, Herbst K, et al. Hiding in the shadows of the HIV epidemic: obesity and hypertension in a rural population with very high HIV prevalence in South Africa. J Hum Hypertens. 2008; 22:263-269.

12. Statistics South Africa. [Accessed 15 November 2009]. http://www.statssa.gov.za

13. Shisana, O.; Rehle, T.; Simbayi, LC.; Zuma, K.; Jooste, S.; Pillayvan-Wyk, V., et al. South African National HIV Prevalence, HIV Incidence, Behaviour and Communication Survey, 2008. Cape Town: HSRC Press; 2009.

14. South African Department of Health. National Antiretroviral Treatment Guidelines. Pretoria: South African Department of Health; 2004. http://www.doh.gov.za/docs/factsheets/guidelines/artguidelines04/sec1.pdf

15. WHO. Report of a WHO Expert Committee. Geneva: WHO; 1995. Physical status: the use and interpretation of anthropometry. http://whqlibdoc.who.int/trs/WHO_TRS_854.pdf

16. StataCorp. Stata Statistical Software: Release 10.1. College Station, TX: Statacorp LP; 2007.

17. Rubin, DB. Multiple imputation for nonresponse in surveys. Hoboken, NJ: John Wiley \& Sons; 1987.

18. Royston P. Multiple imputation of missing values: update of ice. Stata J. 2005; 5:527-536.

19. WHO. Interim WHO clinical staging of HIV/AIDS and HIV/AIDS case definitions for surveillance, African region. Geneva: WHO; 2005. http://www.who.int/hiv/pub/guidelines/clinicalstaging.pdf 
20. Leung CC, Lam TH, Chan WM, Yew WW, Ho KS, Leung G, et al. Lower risk of tuberculosis in obesity. Arch Intern Med. 2007; 167:1297-1304. [PubMed: 17592104]

21. Tverdal A. Body mass index and incidence of tuberculosis. Eur J Respir Dis. 1986; 5:355-362. [PubMed: 3792471]

22. Edwards LB, Livesay VT, Acquaviva FA, Palmer CE. Height, weight, tuberculosis infection and tuberculosis disease. Ach Environ Health. 1971; 22:106-112.

23. Pednekar MS, Hakama M, Hebert JR, Gupta PC. Association of body mass index with all-cause and cause-specific mortality: findings from a prospective cohort study in Mumbai (Bombay), India. Int J Epidemiol. 2008; 37:524-535. [PubMed: 18276634]

24. Abba K, Sudarsanam TD, Grobler L, Volmink J. Nutritional supplements for people being treated for active tuberculosis. Cochrane Database Sys Rev. 2008; 4:CD006086.

25. van Lettow M, Fawzi WW, Semba RD. Triple trouble: the role of malnutrition in tuberculosis and human immunodeficiency virus co-infection. Nutr Rev. 2003; 61:81-90. [PubMed: 12723640]

26. Wanke C. Nutrition and HIV in the international setting. Nutr Clin Care. 2005; 8:44-48. [PubMed: 15850234]

27. Cegielski JP, McMurray DN. The relationship between malnutrition and tuberculosis: evidence from studies in humans and experimental animals. Int J Tuberc Lung Dis. 2005; 8:286-298. [PubMed: 15139466]

28. Kotler DP. Nutritional alterations associated with HIV infection. J Acquir Immune Defic Syndr. 2000; 25 (Suppl 1):S81-S87. [PubMed: 11126432]

29. Salomon J, De TP, Melchior JC. Nutrition and HIV infection. Br J Nutr. 2002; 81 (Suppl 1):S111S119. [PubMed: 11895147]

30. Irlam JH, Visser ME, Siegfried N. Micronutrient supplementation in children and adults with HIV infection. Cochrane Database Syst Rev. 2005; 4:CD003650. [PubMed: 16235333]

31. Chocano-Bedoya P, Ronnenberg AG. Vitamin D and tuberculosis. Nutr Rev. 2009; 67:289-293. [PubMed: 19386033]

32. Badri M, Wilson D, Wood R. Effect of highly active antiretroviral therapy on incidence of tuberculosis in South Africa: a cohort study. Lancet. 2002; 359:2059-2064. [PubMed: 12086758]

33. Fairall LR, Bachmann MO, Louwagie GM, van Vuuren C, Chikobvu P, Steyn D, et al. Effectiveness of antiretroviral treatment in a South African program: a cohort study. Arch Intern Med. 2008; 168:86-93. [PubMed: 18195200]

34. Lawn SD, Myer L, Orrell C, Bekker LG, Wood R. Early mortality among adults accessing a community-based antiretroviral service in South Africa: implications for programme design. AIDS. 2005; 19:2141-2148. [PubMed: 16284464]

35. Braitstein P, Brinkhof MW, Dabis F. Mortality of HIV-1-infected patients in the first year of antiretroviral therapy: comparison between low-income and high-income countries. Lancet. 2006; 367:817-824. [PubMed: 16530575] 

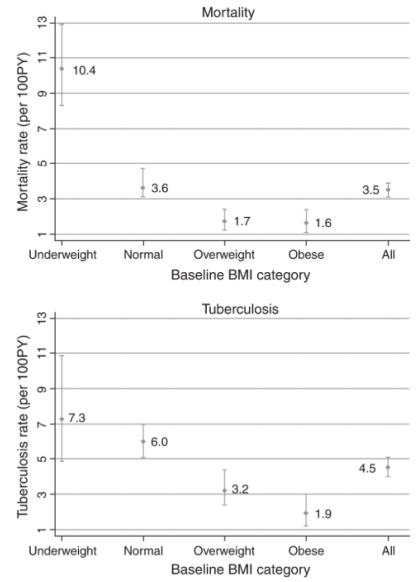

Fig. 1.

Incidence rates of all-cause mortality and tuberculosis among HIV-positive adults in Soweto, South Africa by baseline BMI category. 

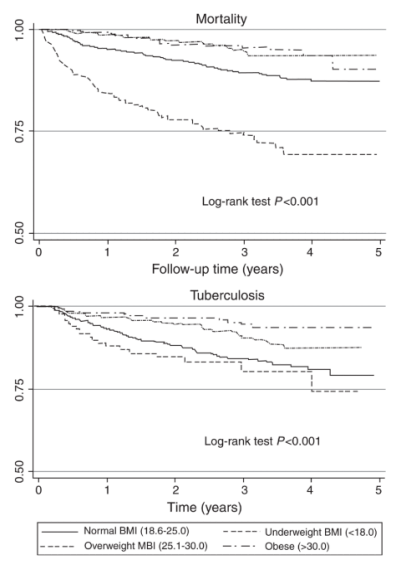

Fig. 2.

Survival among an HIV-infected cohort of adults in Soweto, South Africa to outcomes of all-cause mortality and tuberculosis by baseline BMI category. 
Table 2

Effect of most recent BMI category on all-cause mortality among a cohort of HIV-positive adults in Soweto, South Africa.

\begin{tabular}{|c|c|c|c|c|}
\hline & Univariate HR $(95 \% \mathrm{CI})$ & $P$ & aHR $(95 \%$ CI $)$ & $P$ \\
\hline \multicolumn{5}{|c|}{ Most recent BMI category } \\
\hline Underweight & $3.0(2.2-4.0)$ & $<0.001$ & $1.8(1.3-2.4)$ & $<0.001$ \\
\hline Normal & REF & & REF & \\
\hline Overweight & $0.47(0.32-0.70)$ & $<0.001$ & $0.59(0.40-0.87)$ & 0.008 \\
\hline Obese & $0.36(0.22-0.59)$ & $<0.001$ & $0.48(0.29-0.80)$ & 0.005 \\
\hline HAART use & $0.33(0.22-0.51)$ & $<0.001$ & $0.29(0.19-0.45)$ & $<0.001$ \\
\hline \multicolumn{5}{|c|}{ Most recent CD4 cell count (cells/ $\mu \mathrm{l})$} \\
\hline$<200$ & REF & & REF & \\
\hline $200-349$ & $0.24(0.18-0.35)$ & $<0.001$ & $0.24(0.17-0.35)$ & $<0.001$ \\
\hline $350-500$ & $0.11(0.06-0.20)$ & $<0.001$ & $0.10(0.06-0.20)$ & $<0.001$ \\
\hline$>500$ & $0.09(0.06-0.16)$ & $<0.001$ & $0.12(0.07-0.21)$ & $<0.001$ \\
\hline CPT use & $1.2(1.0-1.6)$ & 0.039 & $0.84(0.65-1.1)$ & 0.180 \\
\hline \multicolumn{5}{|c|}{ Household income (Rand/month) } \\
\hline$<1000$ & REF & & REF & \\
\hline $1001-5000$ & $0.82(0.64-1.0)$ & 0.110 & $0.91(0.72-1.2)$ & 0.475 \\
\hline$>5000$ & $0.94(0.46-1.9)$ & 0.858 & $1.0(0.51-2.1)$ & 0.915 \\
\hline Employed & $1.1(0.85-1.4)$ & 0.569 & $0.80(0.61-1.0)$ & 0.065 \\
\hline Smoking, ever & $1.8(1.4-2.2)$ & $<0.001$ & $1.4(1.0-1.8)$ & 0.024 \\
\hline
\end{tabular}

aHR also adjusted for age, sex, and years since HIV diagnosis. aHR, adjusted hazard ratio; CI, confidence interval; CPT, cotrimoxazole preventive therapy; HR, hazard ratio; REF, reference. 
Table 3

Effect of most recent BMI on incident tuberculosis among a cohort of HIV-positive adults in Soweto, South Africa.

\begin{tabular}{|c|c|c|c|c|}
\hline & Univariate HR (95\% CI) & $P$ & aHR $(95 \% \mathrm{CI})$ & $P$ \\
\hline \multicolumn{5}{|l|}{ BMI category } \\
\hline Underweight & $1.6(1.11-2.34)$ & 0.013 & $1.2(0.82-1.8)$ & 0.332 \\
\hline Normal & REF & & REF & \\
\hline Overweight & $0.55(0.40-0.75)$ & $<0.001$ & $0.62(0.45-0.86)$ & 0.004 \\
\hline Obese & $0.31(0.20-0.48)$ & $<0.001$ & $0.40(0.26-0.63)$ & $<0.001$ \\
\hline HAART use & $0.65(0.49-0.88)$ & 0.004 & $0.44(0.32-0.59)$ & $<0.001$ \\
\hline \multicolumn{5}{|c|}{ CD4 cell count $($ cells $/ \mu \mathrm{l})$} \\
\hline$<200$ & REF & & $\mathrm{REF}$ & \\
\hline $200-349$ & $0.44(0.34-0.60)$ & $<0.001$ & $0.39(0.29-.53)$ & $<0.001$ \\
\hline $350-500$ & $0.34(0.23-0.49)$ & $<0.001$ & $0.30(0.20-0.44)$ & $<0.001$ \\
\hline$>500$ & $0.16(0.10-0.24)$ & $<0.001$ & $0.18(0.11-0.27)$ & $<0.001$ \\
\hline IPT use & $0.71(0.50-1.0)$ & 0.069 & $0.67(0.42-1.1)$ & 0.094 \\
\hline \multicolumn{5}{|c|}{ Household income (Rand/month) } \\
\hline$<1000$ & REF & & REF & \\
\hline $1001-5000$ & $1.0(0.79-1.3)$ & 0.985 & $1.1(0.88-1.4)$ & 0.401 \\
\hline$>5000$ & $0.70(0.29-1.7)$ & 0.439 & $0.82(0.32-1.9)$ & 0.672 \\
\hline Employed & $1.2(0.93-1.5)$ & 0.166 & $1.0(0.77-1.3)$ & 0.906 \\
\hline
\end{tabular}

aHR also adjusted for age, sex, and years since HIV diagnosis. aHR, adjusted hazard ratio; CI, confidence interval; HR, hazard ratio; IPT, isoniazid preventive therapy; REF, reference. 